\title{
Subfoveal choroidal thickness as a predictor of central serous chorioretinopathy
}

V Ambiya ${ }^{1}$, R Yogi ${ }^{1}$, A Li ${ }^{2}$, S Shah ${ }^{3}$, C Sarvaiya ${ }^{3}$, P Mehta ${ }^{4}$, C Meyerele ${ }^{4}, \mathrm{~L}$ Wu ${ }^{5}, \mathrm{R}$ Singh ${ }^{2}$, A Banker ${ }^{3}$ and J Chhablani ${ }^{1}$

\author{
Abstract \\ Purpose The purpose of the study was to \\ evaluate the role of subfoveal choroidal \\ thickness (CT) measurements as a predictor \\ for the course of central serous \\ chorioretinopathy (CSC). \\ Patients and methods Thirty-eight eyes of \\ 33 patients with CSC were analyzed \\ retrospectively. Key inclusion criteria were \\ naive (acute or recurrent) CSC; a minimum of \\ 12 months of follow up; and the availability of \\ good quality enhanced depth imaging SD-OCT \\ images at each visit. Eyes with changes \\ suggestive of chronic CSC and history of any \\ treatment were excluded. Collected data \\ included demographic profile, visual acuity, \\ subfoveal CT, central macular thickness \\ (CMT), and treatment details. Univariate and \\ multivariate analyses for association of \\ baseline features with need for treatment \\ were performed. On the basis of results of \\ this analysis, the 38 eyes were divided into \\ two groups: Group A (subfoveal CT $\leq 356 \mu \mathrm{m}$ ) \\ and Group B (subfoveal CT $>356 \mu \mathrm{m}$ ). \\ Results On univariate analysis, the need for \\ treatment had significantly positive \\ correlation with duration of symptoms \\ $(P=0.02)$, negative correlation with the \\ baseline CT $(P<0.01)$, and no significant \\ correlation with CMT $(P=0.13)$. On \\ multivariate regression analysis, only baseline \\ CT had a statistically significant association \\ with the need for treatment (odds ratio (OR), \\ 0.989; CI, 0.979-0.999; $P=0.048$ ). Group A \\ eyes were more likely to require treatment \\ (Group A: 54.55\%, 12 of 22 eyes; Group B: \\ $18.75 \%$, 3 of 16 eyes; OR: $5.2,95 \%$ CI, \\ 1.15-23.54; $P=0.04)$. There was a significant \\ decrease in subfoveal CT in Group B \\ $(-105.62 \pm 108.91 \mu \mathrm{m} ; P=0.002)$. \\ Conclusion CSC with a subfoveal \\ $\mathrm{CT} \leq 356 \mu \mathrm{m}$ is of chronic nature and is more \\ likely to require treatment rather than \\ observation.
}

Eye (2016) 30, 1623-1629; doi:10.1038/eye.2016.209; published online 30 September 2016

\section{Introduction}

Despite of its high prevalence, central serous chorioretinopathy (CSC) remains poorly understood. Indocyanine green angiography (ICGA) in eyes with CSC demonstrates choroidal vascular abnormalities in the form of abnormally dilated choroidal vasculature in the early phase and choroidal hyperpermeability in the late phase. ${ }^{1-10}$ By using the enhanced depth imaging-optical coherence tomography (EDI-OCT) technique introduced by Spaide et al, 11,12 it has been reported that subfoveal choroidal thickness (CT) is increased in eyes with CSC as compared with normal eyes. ${ }^{13-15}$ The subfoveal choroid in the fellow eyes of patients with unilateral CSC is also thicker than normal eyes, and shows choroidal vascular hyperpermeability on angiography. 16,17

It has been reported that the CT in CSC, which is increased at baseline, gradually decreases over the next 3 months but never reaches the normal level at the end of 1 year. ${ }^{13}$ Thus, the change in $\mathrm{CT}$ in this disorder is well established. However, the role that CT may have in predicting the natural course or management of this condition is still not clear.

In the present study, we wished to evaluate subfoveal CT as a predictor of the course of CSC.

\section{Materials and methods}

A retrospective chart analysis of 50 eyes of 45 patients with naive CSC was done at tertiary eye-care centres from India, United States, and Costa Rica. Institutional review board approval was obtained from each institute for retrospective data collection and analysis. The study adhered to the tenets of the Declaration of Helsinki.

\author{
${ }^{1}$ Srimati Kanuri \\ Santhamma Centre for \\ Vitreo-Retinal Diseases, \\ Hyderabad Eye Research \\ Foundation, LV Prasad Eye \\ Institute, Hyderabad, India \\ ${ }^{2}$ Cole Eye Institute, \\ Cleveland Clinic, \\ Cleveland, OH, USA
}

${ }^{3}$ Banker's Retina Clinic \& Laser Centre, Ahmedabad, India

${ }^{4}$ Retina Division, Wilmer Eye Institute, Johns Hopkins University School of Medicine, Baltimore, MD, USA

\section{${ }^{5}$ Apdo 144-1225 Plaza Mayor, San José, Costa Rica \\ Correspondence: \\ J Chhablani, Srimati Kanuri Santhamma Centre for Vitreo-Retinal Diseases, Hyderabad Eye Research Foundation, LV Prasad Eye Institute, Kallam Anji Reddy campus, Hyderabad 500 034, India Tel: +91 4030612607 ; Fax: +91402354 8271. E-mail: jay.chhablani@ gmail.com}

Received: 17 May 2016 Accepted in revised form: 4 August 2016 Published online: 30 September 2016 
The inclusion criteria were: (i) age $\geq 18$ years; (ii) naive CSC diagnosed by the presence of subretinal fluid (SRF) at the fovea, verified by spectral domain OCT (SD-OCT); (iii) minimum 12 months of follow up; (iv) availability of enhanced depth SD-OCT images (EDI) at each visit. The exclusion criteria were: (i) changes suggestive of chronic CSC such as widespread retinal epithelial atrophic patches; (ii) history of treatment for acute CSC before presentation; (iii) unavailability of EDI SD-OCT scans; (iv) any past vitreoretinal intervention in the form of laser photocoagulation/photodynamic therapy (PDT)/ vitreoretinal surgery/intravitreal injection; (v) any ocular disorder as a cause of vision loss other than CSC; (vi) evidence of glaucoma; (vii) spherical equivalent $\geq \pm 6 \mathrm{D}$; (viii) current steroid therapy for any systemic condition; (ix) cataract surgery in the past 6 months; (x) any media opacity likely to cause attenuation of signal strength in OCT; (xi) history of malignant hypertension or any other systemic disease; (xii) pregnancy.

A detailed ocular history, the demography, laterality, and systemic comorbidities were recorded. The clinical examination included assessment of the best corrected visual acuity (BCVA) in Snellen, spherical equivalent of refractive status of the eye, slit lamp biomicroscopy with a contact lens or non-contact lens, indirect ophthalmoscopy, and digital fundus fluorescein angiography

$(\mathrm{FFA}) \pm$ ICGA, both at the discretion of the clinician.

All eyes were examined with the Heidelberg Spectralis OCT (Heidelberg Engineering, Heidelberg, Germany) or Cirrus HD-OCT (Carl Zeiss Meditec, Dublin, CA, USA) to measure the central macular thickness (CMT) and the subfoveal CT. The subfoveal CT was measured using the EDI-OCT technique previously described by Margolis and Spaide ${ }^{12}$ wherein $6 \mathrm{~mm}$ horizontal and vertical sections, which contained 100 duplicated scans of the macula, going through the foveola were obtained.

The vertical distance between the hyperreflective line of Bruch's membrane and the innermost hyperreflective line of the chorio-scleral interface was taken, and the average of the two scans (vertical and horizontal) was considered as the subfoveal CT. CMT was measured using in-built callipers as a distance between the internal limiting membrane and hyperreflective line of RPE using the scan passing through fovea.

\section{Statistical analysis}

The Snellen BCVA was converted to logarithm of the minimum angle of resolution (logMAR) equivalent for statistical analysis. The change in CT, CMT, and BCVA from baseline to the final outcome was analysed with the Wilcoxon signed-rank test. The association of baseline features with the need for treatment was analysed using univariate and multivariate analyses. The correlation of the CT with CMT, with the duration of symptoms, and with the baseline BCVA was measured using Spearman's Rho test. A univariate analysis was done to correlate the baseline features with the need for treatment $v \mathrm{~s}$ observation, using point biserial correlation coefficient. All the baseline features, which were found to have a significant correlation, were further analysed using multiple logistic regression, and the association was shown as odds ratio (OR) with $95 \%$ confidence intervals (CI).

The average subfoveal CT of eyes that required treatment was compared with that of the eyes that were observed without intervention until the last follow up. The average of the adjoining $95 \% \mathrm{CI}$ limits of the two means was hypothesized as the critical CT, ' $X^{\prime} \mu \mathrm{m}$, to decide if an eye needed treatment or not. Thereafter, the 38 eyes were divided into two groups: Group A having $\mathrm{CT} \leq \mathrm{X} \mu \mathrm{m}$; Group B having $\mathrm{CT}>\mathrm{X} \mu \mathrm{m}$. The various clinical features, outcomes, and association with treatment were compared between the two groups. The numerical variables between Groups A and B were analysed using an unpaired $t$-test. The association of categorical variables with the two groups was calculated in the form of $\mathrm{OR}$, and the significance analysed using Fisher's exact test.

All measurements taken with the Spectralis scanner were converted to Cirrus measurements by adding $19.23 \mu \mathrm{m}$, consistent with the normalization proposed by Lammer et al. ${ }^{18}$

A $P$-value of $<0.05$ was considered as statistically significant.

\section{Results}

Fifty eyes of 45 patients with treatment-naive CSC with minimum follow up of 1 year were evaluated. Twelve eyes were excluded due to unavailability of good quality EDI images. The present study included 38 eyes of 33 patients (20 Indians and 18 Caucasians), with a mean age of $44.3 \pm 8.8$ years. Twenty-six were men and seven were women. Table 1 summarizes the baseline and follow-up characteristics of the 38 eyes.

At baseline all eyes were phakic with a mean CMT of $403.43 \pm 143.97 \mu \mathrm{m}$, a mean subfoveal CT of $378.89 \pm 147.82 \mu \mathrm{m}$, and a history of prior exposure to systemic steroids in $21.05 \%$ (8 of 38 ) of cases. FFA was done in 22 of 38 eyes and showed a single leak in 13 cases, and more than one leak in 9 cases. Out of 38 eyes, 27 eyes were observed at the baseline. However, out of these 27, 5 eyes underwent treatment during follow up (PDT in two, laser photocoagulation in three eyes). At baseline, 11 eyes were treated with focal laser photocoagulation considering their history. Overall, six laser-treated eyes required re-treatment during the follow-up period (laser 
Table 1 Overall features of 38 eyes at baseline and on final follow-up visit. Univariate analysis of correlation of baseline features history of treatment during follow up

\begin{tabular}{|c|c|c|c|}
\hline Overall baseline/final features & $\begin{array}{c}\mathrm{N}=38 \text { eyes of } 33 \\
\text { patients }\end{array}$ & $\begin{array}{l}\text { Univariate analysis (point biserial } \\
\text { correlation) }\end{array}$ & $\begin{array}{c}\text { Multivariate logistic regression } \\
\text { analysis }\end{array}$ \\
\hline Age (years $\pm \mathrm{SD})$ & $44.26 \pm 8.77$ & $r_{\mathrm{pb}}=0.11 ; P=0.53$ & - \\
\hline Male: female $(n)$ & $26: 7$ & - & - \\
\hline Spherical equivalent $(\mathrm{D} \pm \mathrm{SD})$ & $+0.62 \pm 0.93$ & - & - \\
\hline Steroid exposure $(n, \%)$ & $8(21.05 \%)$ & - & - \\
\hline Duration of symptoms (days $\pm S D$ ) & $59.74 \pm 121.69$ & $r_{\mathrm{pb}}=0.4 ; P<0.01^{\mathrm{a}}$ & $\begin{array}{c}\mathrm{OR}=1.009(0.995-1.022) \\
P=0.20\end{array}$ \\
\hline Baseline BCVA (Snellen \pm SD logMAR) & $20 / 29.5 \pm 0.18$ & $r_{\mathrm{pb}}=-0.1 ; P=0.56$ & - \\
\hline RPE changes at baseline $(n, \%)$ & $8(21.05 \%)$ & & - \\
\hline Baseline CMT $(\mu \mathrm{m} \pm \mathrm{SD})$ & $403.43 \pm 143.97$ & $r_{\mathrm{pb}}=-0.26 ; P=0.13$ & - \\
\hline Baseline CT $(\mu \mathrm{m} \pm \mathrm{SD})$ & $378.89 \pm 147.82$ & $r_{\mathrm{pb}}=-0.43 ; P<0.01^{\mathrm{a}}$ & $\begin{array}{c}\mathrm{OR}=0.989(0.979-0.999) \\
P=0.048^{\mathrm{a}}\end{array}$ \\
\hline Duration of follow up (days $\pm S D$ ) & $23 \pm 12.90$ & - & - \\
\hline Final BCVA (Snellen \pm SD logMAR) & $20 / 36 \pm 0.35(P=0.55)$ & - & - \\
\hline Final CMT $(\mu \mathrm{m} \pm \mathrm{SD})$ & $\begin{array}{c}255.43 \pm 79.52 \\
(P=0.03)^{\mathrm{a}}\end{array}$ & - & - \\
\hline Final CT $(\mu \mathrm{m} \pm \mathrm{SD})$ & $\begin{array}{c}341.9 \pm 119.41 \\
(P<0.001)^{\mathrm{a}}\end{array}$ & - & - \\
\hline
\end{tabular}

Abbreviations: BCVA, best corrected visual acuity; CMT, central subfield retinal thickness; CT, subfoveal choroidal thickness; OR, odds ratio; rpb, point biserial coefficient. ${ }^{\text {a }}$ Statistically significant.

$P$-values have been calculated in comparison with baseline.

photocoagulation in five, PDT in one). After a mean follow-up duration of $23 \pm 12.90$ months, the final mean BCVA was $0.25 \pm 0.35 \log$ MAR $(20 / 36$ Snellen; $P=0.55$, as compared with baseline BCVA), with a statistically significant decrease in the mean CMT to $255.43 \pm 79.52 \mu \mathrm{m}$ $(P=0.03)$ and also in the mean subfoveal CT to $341.9 \pm 119.41 \mu \mathrm{m}(P<0.001)$. Representative cases are shown as Figure 1.

The baseline subfoveal CT had a statistically significant negative correlation with the duration of symptoms $(R=-0.377 ; P=0.03)$ and positive correlation with the baseline CMT $(R=0.524 ; P<0.001)$, but no significant correlation with the baseline BCVA $(R=-0.035 ; P=0.83)$, final BCVA $(R=-0.187 ; P=0.31)$, or with age $(R=-0.15$; $P=0.37)$.

On univariate analysis, we found that the need for treatment had a statistically significant positive correlation with the duration of symptoms $(P=0.02)$, a significant negative correlation with the baseline CT $(P<0.01)$, but no significant correlation with age $(P=0.53)$, baseline BCVA $(P=0.56)$, or with CMT $(P=0.13)$. (Table 1$)$ On multivariate regression analysis of these two significant variables (CT and duration of symptoms), we found that the need for treatment had a statistically significant association only with baseline CT $(P=0.04)$ and not with the duration of symptoms $(P=0.20)$. (Table 1$)$

In agreement with the results of multiple regression, we found the mean baseline subfoveal CT to be significantly lower in the eyes that required treatment with laser photocoagulation/PDT/both (307.07 $\mu \mathrm{m}$; 95\% CI, 257.82-
$356.31 \mu \mathrm{m})$, as compared with the eyes that were observed without treatment $(420.48 \mu \mathrm{m}$; 95\% CI, 356.13-484.82 $\mu \mathrm{m}$; $P=0.009$ ). (Figure 2) We found $356 \mu \mathrm{m}$ to be the overlapping whole number between the adjoining 95\% CI limits of the mean CT in the two groups, and hypothesized it to be a possible critical value of CT to decide for treating or observing the eyes with CSC. We, therefore, divided the 38 eyes into two groups: Group A $(\mathrm{CT} \leq 356 \mu \mathrm{m})$, Group B (CT $>356 \mu \mathrm{m})$ and did a comparative analysis of the features of the two groups.

The baseline and final characteristics of the eyes in Groups A and B are summarized in Table 2. There were 22 eyes in Group A and 16 eyes in Group B, with no statistically significant difference in the age $(P=0.14)$, sex $(P=0.70)$, baseline visual acuity $(P=0.34)$, and the spherical equivalent $(P=0.71)$ between the two groups. Group A had a statistically significantly lower mean subfoveal CT (Group A: 274.68 $\pm 47.18 \mu \mathrm{m}$; Group B: $522.19 \pm 113.82 \mu \mathrm{m} ; P<0.001)$ and also a lower mean CMT (Group A: $336.77 \pm 112.39 \mu \mathrm{m}$; Group B:

$501.2 \pm 130.86 \mu \mathrm{m} ; P<0.001)$ as compared with Group B. Treatment in the form of laser photocoagulation or PDT was done in $54.55 \%$ (12 of 22) eyes in Group A vs 18.75\% (3 of 16) eyes in Group B, with an OR of 5.2 (95\% CI, 1.1523.54; $P=0.04)$. There was no statistically significant difference between the final BCVA in the two groups $(P=0.76)$. The rate of recurrence of SRF was not significantly different in the two groups (OR, 1.83; 95\% CI, $0.48-7.07 ; P=0.51$ ). There was a trend towards more likelihood of RPE changes occurring in Group A as compared with Group B (OR, 7; 95\% CI, 0.76-64.08; 
$P=0.11)$. There was no significant decrease in subfoveal CT in Group A $(+6.40 \pm 61.50 \mu \mathrm{m} ; P=1)$, but statistically significant decrease in Group B $(-105.62 \pm 108.91 \mu \mathrm{m}$; $P=0.002)$. The final subfoveal CT was significantly lower in Group A $(282.69 \pm 59.94 \mu \mathrm{m})$ as compared with Group B $(430.71 \pm 132.70 \mu \mathrm{m} ; P<0.001)$. The CMT decreased significantly in both groups $(P<0.001)$, and the decrease was significantly more in Group B $(P=0.01)$.

\section{Discussion}

Although ICGA has proven the role of choroidal vascular abnormalities in CSC, it was only after the advent of EDI-OCT ${ }^{11}$ that the knowledge of $\mathrm{CT}$ gained importance in understanding the course of the disease. CT is known to be increased in eyes with CSC. ${ }^{13-15}$ It gradually decreases over the next 3 months. ${ }^{13}$ The CT is greater in areas with leakage on FFA, and in areas with choroidal vascular hyperpermeability and punctate hyperfluorescent spots on ICGA, than in the unaffected areas. ${ }^{19}$ The CT, although not significantly affected by laser photocoagulation, significantly reduces after PDT and is associated with decreased choroidal vascular hyperpermeability on ICGA. ${ }^{15}$
Our study highlights the importance of choroidal imaging in inferring the stage and the possible role in deciding the management of CSC. We found that subfoveal CT has a statistically significant negative correlation with the duration of symptoms, and is significantly higher in patients with a shorter duration of symptoms. This is contrary to the findings of Kim et al, ${ }^{16}$ who reported no correlation of CT with symptom

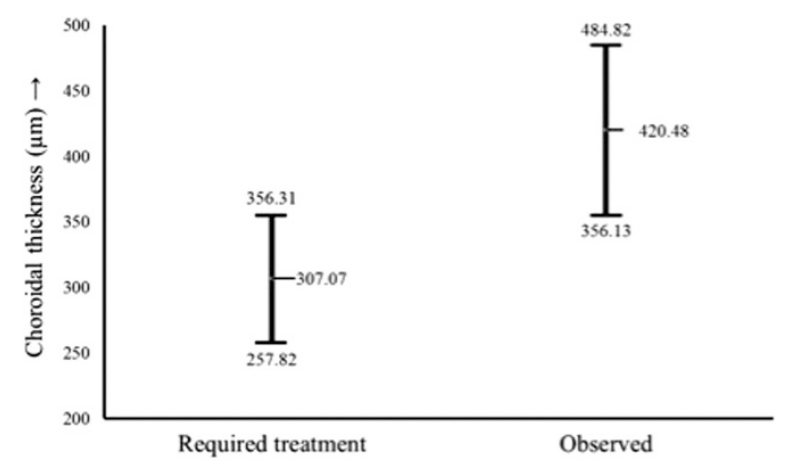

Figure 2 Baseline subfoveal choroidal thickness (with 95\% CI) of eyes that required treatment $(n=16)$ vs eyes that were observed $(n=22)$.

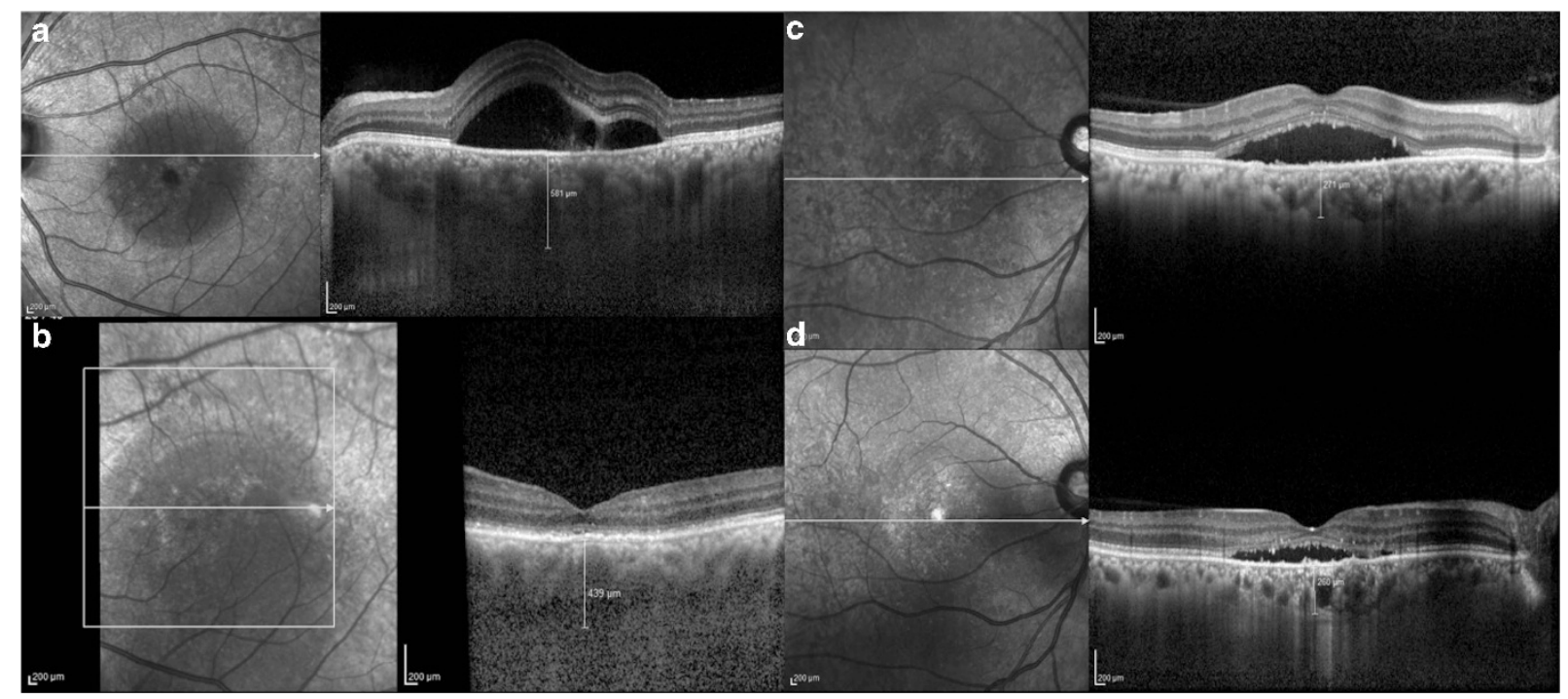

Figure 1 A 43-year-old man presented with blurring of vision in his right eye since 20 days. He had a history of similar episode 4 months ago which resolved spontaneously. His BCVA was 20/60 in his left eye. On clinical examination including fluorescein angiography and SD-OCT (a), a diagnosis of central serous chorioretinopathy was made. At baseline his CMT and subfoveal choroidal thickness (SFCT) were 678 and 581 microns, respectively. In view of it being the first episode, the patient was observed without any intervention. He was on regular follow up and subretinal fluid resolved completely at third month follow up. At his last follow up, at 36 months, his BCVA was maintained at 20/30 with no fluid on SD-OCT (b) with CMT and SFCT of 206 and 238 microns, respectively. A 30-year-old man presented with blurring of vision in his right eye since 20 days. He had no history of similar episode in the past. His BCVA was 20/20 in his both eyes. On clinical examination including fluorescein angiography and SD-OCT (c), diagnosis of recurrent central serous chorioretinopathy was made. At baseline his CMT and SFCT were 440 and 271 microns, respectively. In view of recurrent nature of disease, the patient was advised laser photocoagulation to the leak seen on fluorescein angiography. He, however, did not give consent for treatment. He did not receive any treatment till his last follow up at 13 months, when his BCVA had dropped to 20/63 with persistent fluid on SD-OCT (d), with CMT and SFCT of 281 and 260 microns, respectively. 
Table 2 Baseline and final characteristics of eyes in groups A and B

\begin{tabular}{|c|c|c|c|}
\hline Feature & $\begin{array}{c}\text { Group A }(C T \leq 356 \mu m) \\
N=22\end{array}$ & $\begin{array}{c}\text { Group B }(C T>356 \mu m) \\
N=16\end{array}$ & P-value \\
\hline Age (years $\pm S D)$ & $46.05 \pm 9.66$ & $41.81 \pm 6.92$ & 0.14 \\
\hline Male: female $(n)$ & $18: 4$ & $12: 4$ & 0.70 \\
\hline Spherical equivalent $(\mathrm{D} \pm \mathrm{SD})$ & $0.61 \pm 0.94$ & $0.75 \pm 0.79$ & 0.71 \\
\hline Steroid exposure $(n, \%)$ & $6(27.28 \%)$ & $2(12.5 \%)$ & 0.43 \\
\hline Duration of symptoms (days \pm SD) & $83.8 \pm 121.84$ & $15.27 \pm 11.22$ & $0.04^{\mathrm{a}}$ \\
\hline Baseline BCVA (Snellen \pm SD logMAR) & $20 / 28 \pm 0.154$ & $20 / 32 \pm 0.214$ & 0.52 \\
\hline RPE changes $(n, \%)$ & $7(31.82 \%)$ & $1(6.25 \%)$ & - \\
\hline Baseline CMT $(\mu \mathrm{m} \pm \mathrm{SD})$ & $336.77 \pm 112.39$ & $501.2 \pm 130.86$ & $<0.001^{\mathrm{a}}$ \\
\hline Baseline CT $(\mu \mathrm{m} \pm \mathrm{SD})$ & $274.68 \pm 47.18$ & $522.19 \pm 113.82$ & $<0.001^{\mathrm{a}}$ \\
\hline Treatment given & $12(54.55 \%)$ & $3(18.75 \%)$ & - \\
\hline Final BCVA (Snellen \pm SD logMAR) & $20 / 37 \pm 0.37$ & $20 / 34 \pm 0.33$ & 0.76 \\
\hline Recurrence & $10(45.45 \%)$ & $5(31.25 \%)$ & - \\
\hline Final CMT $(\mu \mathrm{m} \pm \mathrm{SD})$ & $237.19 \pm 70.61$ & $279.38 \pm 86.33$ & 0.11 \\
\hline Final CT $(\mu \mathrm{m} \pm \mathrm{SD})$ & $282.69 \pm 59.94$ & $430.71 \pm 132.70$ & $<0.001^{\mathrm{a}}$ \\
\hline Decrease in CMT $(\mu \mathrm{m} \pm \mathrm{SD})$ & $107.24 \pm 114.45$ & $232.46 \pm 156.31$ & $\begin{array}{l}<0.001^{\mathrm{a}}(\mathrm{A}) \\
<0.001^{\mathrm{a}}(\mathrm{B})\end{array}$ \\
\hline Decrease in CT $(\mu \mathrm{m} \pm \mathrm{SD})$ & $-6.40 \pm 61.50$ & $105.62 \pm 108.91$ & $\begin{array}{c}0.01^{\mathrm{a}}(\mathrm{A} \text { vs } \mathrm{B}) \\
1.0(\mathrm{~A}) \\
0.002^{\mathrm{a}}(\mathrm{B}) \\
<0.001^{\mathrm{a}}(\mathrm{A} \text { vs } \mathrm{B})\end{array}$ \\
\hline
\end{tabular}

Abbreviations: BCVA, best corrected visual acuity; CMT, central subfield retinal thickness; CT, subfoveal choroidal thickness; D, dioptres;

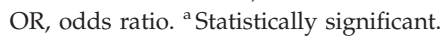

duration in their series of cases. We used our findings to hypothesize that eyes with thicker choroid are in the acute stage of CSC; have a better visual potential and can be observed. In contrast, the eyes with a thinner choroid at baseline are going into a chronic stage, which may require treatment in the form of laser photocoagulation or PDT. A similar correlation of CT with final outcome has also been reported with other ocular conditions. Kang et al ${ }^{20}$ reported that eyes with a thicker choroid in cases of typical exudative age-related macular degeneration responded better to intravitreal ranibizumab injections, and had better potential for visual recovery because of more abundant choroidal blood supply and choriocapillaris. The CT in myopia also has a positive correlation with final visual acuity. ${ }^{21,22}$ In polypoidal choroidal vasculopathy, ${ }^{23}$ Vogt-Koyanagi-Harada disease ${ }^{24}$ and Behçet's disease, ${ }^{25}$ the choroid is thickened during the active phase and thereafter shows progressive thinning. The CT, therefore, has the potential to be used as a parameter to monitor the activity of these diseases.

In our current series, we calculated that a CT of $356 \mu \mathrm{m}$ was the critical number to decide if an eye was more likely to require treatment or not. The eyes that had a $\mathrm{CT} \leq 356 \mu \mathrm{m}$ (Group A) had a significantly longer duration of symptoms, suggestive of a chronic nature, as compared with the eyes with a thicker choroid ( $>356 \mu \mathrm{m}$, Group B). The eyes in Group A had approximately five times the odds of requiring treatment as compared with Group B. The eyes in Group A had approximately seven times the odds of developing RPE changes as compared with Group B, suggestive of chronicity, even though the ratio did not have statistical significance. Unlike the significant decrease in CT seen in Group B, Group A did not show a significant change in the CT at the end of follow up, which is again a sign of the acute nature of Group B.

Observation has been the mainstay of treatment of CSC in patients who present with a good visual acuity and symptom duration of $<3$ months, after which treatment is recommended. ${ }^{26,27}$ So one could question the role of monitoring CT vs duration of symptoms as an indicator for treatment of CSC. It is known that subfoveal CT is higher in the acute stage and gradually decreases during the course of CSC. ${ }^{13}$ Unlike the duration of symptoms, which is a subjective parameter, we propose that subfoveal CT, being an objective parameter, may be an indicator of the activity of the disease. However, we cannot conclude from the small number of study eyes that CT can be used as a biomarker for the treatment of CSC. Moreover, eyes with loss of ellipsoid layer are unlikely to gain visually from any intervention, irrespective of the CT measurement.

The strength of our study is the long follow up of almost 2 years; the treatment naivety of the study eyes and the exclusion of eyes with chronic CSC. However, limitations inherent in retrospective studies cannot be overlooked. Being a retrospective and a multicentric study, there was no standard protocol for initiating 
treatment. There is a possibility of ethnic variability in the course of the disease, which could have affected our results. We could not evaluate different treatment modalities with respect to the $\mathrm{CT}$, therefore, we cannot provide a treatment algorithm based on CT. Another limitation is the non-availability of the technology in many clinics; in addition, there is no final agreement on the accuracy and the uniformity of CT measurements.

In conclusion, choroidal thickness measurement on EDI-OCT could be a potential criterion for monitoring the disease activity. In present study, eyes with a relatively thinner choroid with persistent fluid at presentation required treatment more often compared with the eyes with thicker choroid at presentation. Future trials on the role of choroidal thickness as a guide for various interventions may provide a better insight in the management of this disease.

\section{Summary}

What was known before

- Choroidal thickness increases in central serous chorioretinopathy.

- Choroidal thickness in central serous chorioretinopathy gradually decreases over the next 3 months, but never reaches the normal level at the end of 1 year.

What this study adds

- Choroidal thickness measurement on EDI-OCT could be a potential criterion for monitoring the disease activity.

\section{Conflict of interest}

The authors declare no conflict of interest.

\section{References}

1 Tsujikawa A, Ojima Y, Yamashiro K, Ooto S, Tamura H, Nakagawa $S$ et al. Punctate hyperfluorescent spots associated with central serous chorioretinopathy as seen on indocyanine green angiography. Retina 2010; 30(5): 801-809.

2 Spaide RF, Hall L, Haas A, Campeas L, Yannuzzi LA, Fisher YL et al. Indocyanine green videoangiography of older patients with central serous chorioretinopathy. Retina 1996; 16(3): 203-213.

3 Scheider A, Nasemann JE, Lund OE. Fluorescein and indocyanine green angiographies of central serous choroidopathy by scanning laser ophthalmoscopy. Am J Ophthalmol 1993; 115(1): 50-56.

4 Prunte C, Flammer J. Choroidal capillary and venous congestion in central serous chorioretinopathy. Am J Ophthalmol 1996; 121(1): 26-34.

5 Prunte $\mathrm{C}$. Indocyanine green angiographic findings in central serous chorioretinopathy. Int Ophthalmol 1995; 19(2): 77-82.
6 Piccolino FC, Borgia L. Central serous chorioretinopathy and indocyanine green angiography. Retina 1994; 14(3): 231-242.

7 Menchini U, Virgili G, Lanzetta P, Ferrari E. Indocyanine green angiography in central serous chorioretinopathy. ICG angiography in CSC. Int Ophthalmol 1997; 21(2): 57-69.

8 Kitaya N, Nagaoka T, Hikichi T, Sugawara R, Fukui K, Ishiko $\mathrm{S}$ et al. Features of abnormal choroidal circulation in central serous chorioretinopathy. Br J Ophthalmol 2003; 87(6): 709-712.

9 Guyer DR, Yannuzzi LA, Slakter JS, Sorenson JA, Ho A, Orlock D. Digital indocyanine green videoangiography of central serous chorioretinopathy. Arch Ophthalmol 1994; 112(8): 1057-1062.

10 Piccolino FC, Borgia L, Zinicola E, Zingirian M. Indocyanine green angiographic findings in central serous chorioretinopathy. Eye 1995; 9(Pt 3): 324-332.

11 Spaide RF, Koizumi H, Pozzoni MC. Enhanced depth imaging spectral-domain optical coherence tomography. Am J Ophthalmol 2008; 146(4): 496-500.

12 Margolis R, Spaide RF. A pilot study of enhanced depth imaging optical coherence tomography of the choroid in normal eyes. Am J Ophthalmol 2009; 147(5): 811-815.

13 Brandl C, Helbig H, Gamulescu MA. Choroidal thickness measurements during central serous chorioretinopathy treatment. Int Ophthalmol 2014; 34(1): 7-13.

14 Imamura Y, Fujiwara T, Margolis R, Spaide RF. Enhanced depth imaging optical coherence tomography of the choroid in central serous chorioretinopathy. Retina 2009; 29(10): 1469-1473.

15 Maruko I, Iida T, Sugano Y, Ojima A, Ogasawara M, Spaide RF. Subfoveal choroidal thickness after treatment of central serous chorioretinopathy. Ophthalmology 2010; 117(9): 1792-1799.

16 Kim YT, Kang SW, Bai KH. Choroidal thickness in both eyes of patients with unilaterally active central serous chorioretinopathy. Eye 2011; 25(12): 1635-1640.

17 Maruko I, Iida T, Sugano Y, Ojima A, Sekiryu T. Subfoveal choroidal thickness in fellow eyes of patients with central serous chorioretinopathy. Retina 2011; 31(8): 1603-1608.

18 Lammer J, Scholda C, Prunte C, Benesch T, Schmidt-Erfurth U, Bolz M. Retinal thickness and volume measurements in diabetic macular edema: a comparison of four optical coherence tomography systems. Retina 2011; 31(1): 48-55.

19 Jirarattanasopa P, Ooto S, Tsujikawa A, Yamashiro K, Hangai M, Hirata $\mathrm{M}$ et al. Assessment of macular choroidal thickness by optical coherence tomography and angiographic changes in central serous chorioretinopathy. Ophthalmology 2012; 119(8): 1666-1678.

20 Kang HM, Kwon HJ, Yi JH, Lee CS, Lee SC. Subfoveal choroidal thickness as a potential predictor of visual outcome and treatment response after intravitreal ranibizumab injections for typical exudative age-related macular degeneration. Am J Ophthalmol 2014; 157(5): 1013-1021.

21 Nishida Y, Fujiwara T, Imamura Y, Lima LH, Kurosaka D, Spaide RF. Choroidal thickness and visual acuity in highly myopic eyes. Retina 2012; 32(7): 1229-1236.

22 Wang NK, Lai CC, Chu HY, Chen YP, Chen KJ, Wu WC et al. Classification of early dry-type myopic maculopathy with macular choroidal thickness. Am J Ophthalmol 2012; 153(4): 669-677, 677 e661-662.

23 Yang LH, Jonas JB, Wei WB. Optical coherence tomographic enhanced depth imaging of polypoidal choroidal vasculopathy. Retina 2013; 33(8): 1584-1589. 
24 da Silva FT, Sakata VM, Nakashima A, Hirata CE, Olivalves E, Takahashi WY et al. Enhanced depth imaging optical coherence tomography in long-standing Vogt-Koyanagi-Harada disease. Br J Ophthalmol 2013; 97(1): 70-74.

25 Kim M, Kim H, Kwon HJ, Kim SS, Koh HJ, Lee SC. Choroidal thickness in Behcet's uveitis: an enhanced depth imaging-optical coherence tomography and its association with angiographic changes. Invest Ophthalmol Vis Sci 2013; 54(9): 6033-6039.

26 Ross A, Ross AH, Mohamed Q. Review and update of central serous chorioretinopathy. Curr Opin Ophthalmol 2011; 22(3): 166-173.

27 Wang MS, Sander B, Larsen M. Retinal atrophy in idiopathic central serous chorioretinopathy. Am J Ophthalmol 2002; 133(6): 787-793. 